\title{
Comparison of effects of thrombolytic therapy and primary percutaneous coronary intervention in elderly patients with acute ST-segment elevation myocardial infarction on in-hospital, six-month, and one-year mortality
}

Ibrahim Kocayigit ${ }^{1}$, Selcuk Yaylaci², Altug Osken ${ }^{3}$, Ercan Aydın $^{4}$, Salih Sahinkus ${ }^{1}$, Yusuf Can ${ }^{1}$, Ahmed Bilal Genc ${ }^{2}$, Huseyin Gunduz ${ }^{1}$

\author{
'Department of Cardiology, Sakarya University Education and Research Hospital, \\ Sakarya, Turkey \\ 2Department of Internal Medicine, Sakarya University Education and Research \\ Hospital, Sakarya, Turkey \\ ${ }^{3}$ Department of Cardiology, Dr. Siyami Ersek Cardiovascular and Thoracic Surgery \\ Centre, Istanbul, Turkey \\ ${ }^{4}$ Department of Cardiology, Vakfikebir State Hospital, Trabzon, Turkey
}

Submitted: 9 February 2019

Accepted: 18 April 2019

Arch Med Sci Atheroscler Dis 2019; 4: e82-e88

DOI: https://doi.org/10.5114/amsad.2019.85378

Copyright @ 2019 Termedia \& Banach

\begin{abstract}
Introduction: This study aims to evaluate the effect of primary percutaneous coronary intervention ( $\mathrm{PCI}$ ) and thrombolytic therapy (TT) on the in-hospital adverse events, in-hospital and long-term mortality in patients over 65 years of age with acute ST-segment elevation myocardial infarction (STEMI). Material and methods: A total of 111 retrospectively screened patients (73 males, mean age: $73.4 \pm 5.9$ years) over 65 years of age with STEMI, who underwent TT or primary $\mathrm{PCl}$, were included in the study. Patients' characteristics, in-hospital outcomes, and 6-month and 1-year mortalities were recorded. Results: Our study was conducted with 111 patients over 65 years of age with STEMI (73 males, 38 females). Of the patients, 66 (59.5\%) were treated with thrombolytics, and 45 (40.5\%) patients underwent primary $\mathrm{PCl}$. Door-toneedle time was $25.9 \pm 7.8 \mathrm{~min}$ in the TT group, whereas door-to-balloon time was $84.4 \pm 20.0 \mathrm{~min}$ in the $\mathrm{PCl}$ group. Time from symptom onset to hospital admission was $213.6 \pm 158.4 \mathrm{~min}$ in the thrombolytic group, and $166.8 \pm 112.8$ min in the $\mathrm{PCl}$ group. Rescue $\mathrm{PCl}$ was performed in 7 (10.6\%) patients in the TT group due to lack of reperfusion. Recurrent infarction was observed in 5 (7.6\%) patients in the TT group and in $2(4.4 \%)$ patients in the $\mathrm{PCl}$ group. Non-haemorrhagic stroke was observed in $1(1.5 \%)$ patient in the thrombolytic-administered group and in $4(8.9 \%)$ patients in the $\mathrm{PCl}$ group. No intracranial haemorrhage was observed in any patient. Major haemorrhage was observed in $4(6.1 \%)$ patients in the TT group and in $4(8.9 \%)$ patients in the $\mathrm{PCl}$ group. Six-month and 1-year mortalities were present in $15(22.7 \%)$ patients and 19 patients in thrombolytic group, and $8(17.8 \%)$ and $8(17.8 \%)$ patients in the $\mathrm{PCl}$ group, respectively. Binary logistic regression analysis indicated that the patient's age was the only predictor for 1 -year mortality (odds ratio $(O R)=$ $1.1,95 \%$ confidence interval $(\mathrm{Cl}): 1.019-1.188, p=0.015)$.

Conclusions: Considering the in-hospital adverse outcomes, in-hospital mortality, and 6-month mortality rates, TT and primary $\mathrm{PCI}$ have similar effects in STEMI patients aged 65 years and over according to the results of our study. Although 1-year mortality was higher in the TT group, it was not statistically significant.
\end{abstract}

Key words: percutaneous coronary intervention, thrombolytic therapy, elderly patients, ST-segment elevation myocardial infarction.

\author{
Corresponding author: \\ Ibrahim Kocayigit \\ Department \\ of Cardiology \\ Sakarya University \\ Education and \\ Research Hospital \\ Sakarya, Turkey \\ Phone: +90 5309323075 \\ E-mail: ikocayigit@gmail.com
}




\section{Introduction}

Acute myocardial infarction (AMI) is a common clinical syndrome with high morbidity and mortality throughout the world [1]. ST-segment elevation myocardial infarction (STEMI) is characterised by persistent ST elevation in electrocardiogram (ECG) or characteristic symptoms of myocardial ischaemia accompanied by newly developed left branch block and increased biomarkers associated with myocardial necrosis [1]. Today, $25 \%$ to $40 \%$ of patients presenting with myocardial infarction are known to have STEMI [2]. In recent years, about 5-6\% in-hospital and 7-18\% 1-year STEMI-related mortality rates are decreasing thanks to the interventions and drug treatment in accordance with current guidelines [3].

Early diagnosis and rapid reperfusion are essential in STEMI treatment. Delay in reperfusion has been found to be associated with increased mortality and morbidity [4]. Thrombolytic therapy (TT) or primary percutaneous coronary intervention $(\mathrm{PCl})$ is used to restore patency and re-flow of blood in the coronary artery occluded by thrombus during STEMI. The main goal of the reperfusion is to reduce the time of total ischaemia by appropriate and timely treatment [5]. Some performance measures such as door-to-needle time and doorto-balloon time have been developed to assess the duration of total ischaemia. Today, the reperfusion period is prolonged due to the lack of primary $\mathrm{PCl}$ in many hospitals in developing countries, which negatively affects in-hospital and long-term mortality. In cases where primary $\mathrm{PCl}$ is not available, TT should be started without delay [1].

Acute myocardial infarction is more common in the elderly, in whom it has higher mortality and morbidity compared to that seen in the young population. Of the patients admitted to hospitals due to STEMI, $40-50 \%$ are composed of people aged 65 years and older [6]. More than $60 \%$ of myocardial infarction-related deaths occur in this age group [7]. In many studies, primary $\mathrm{PCl}$ has been found to be superior to TT in achieving successful reperfusion [8]. Primary $\mathrm{PCl}$ is superior to TT especially in elderly patients in terms of reperfusion success [9]. For this reason, there is a tendency to perform $\mathrm{PCl}$ in elderly patients with STEMI, but many of the elderly patients with STEMI are treated with TT because there is still no possibility of 24-hour primary $\mathrm{PCl}$ in many centres in developing countries.

Our aim is to compare the in-hospital major bleeding, stroke, and mortality rates and 6-month and 1-year mortality rates in elderly patients with STEMI and receiving TT and primary PCI treatment. We investigated the effect of TT on the in-hospital and long-term outcome, especially in elderly patients, compared to the primary $\mathrm{PCl}$ because many hospitals in developing countries do not have 24hour primary $\mathrm{PCl}$.

\section{Material and methods}

Our study was performed retrospectively by screening the files of the patients over 65 years of age who underwent TT or primary $\mathrm{PCl}$ between 2010 and 2013 with the diagnosis of acute STEMI and followed up at the Sakarya University Training and Research Hospital Department of Cardiology Coronary Intensive Care Unit. Approval of the Sakarya University Medical Faculty Ethics Committee was obtained for the study protocol. The inclusion criteria of the study were as follows: Patients with persistent chest discomfort or other symptoms suggestive of ischaemia and ST-segment elevation in at least two contiguous leads as STEMI, treated with TT or primary $\mathrm{PCl}$, and over 65 years of age. The exclusion criteria were: Patients admitted at the subacute stage (symptom onset-to-admission $>12 \mathrm{~h}$ ), patients who underwent cardiac arrest as a result of $A M I$ and were followed up under mechanical ventilation, and patients admitted with cardiogenic shock manifestations.

The patients' records were accessed from the hospital records, and the patients' age, gender, hypertension, diabetes mellitus, smoking status, coronary artery disease history, door-to-balloon and door-to-needle times, lipid profile, urea, creatinine, haemogram, haematocrit, and blood glucose values were obtained. STEMI localisation was determined according to the ECG records taken at the time of admission.

Because there was no invasive catheter laboratory that was active $24 \mathrm{~h}$ a day in our hospital at that time, appropriate patients with STEMI admitted outside of working hours received TT. After administering acetylsalicylic acid and concomitant treatment with clopidogrel in the emergency department to the patients who would receive TT, they were taken into the coronary intensive care unit for thrombolytic therapy. After questioning about contraindications to thrombolytics, streptokinase or alteplase was administered intravenously to the appropriate patients. After thrombolysis, patients were evaluated for reperfusion. Non-reperfused patients were taken to the catheter laboratory for rescue $\mathrm{PCl}$. Early coronary angiography was performed to the patients following TT. Only three of the 66 patients did not undergo coronary angiography because the patient and relatives did not accept the procedure. All the patients who were to undergo primary percutaneous intervention were taken to the catheter laboratory after administering acetylsalicylic acid and concomitant treatment with clopidogrel in the emergency department, and primary PCI (balloon angioplasty and/or stent implantation) was 
performed on the infarcted coronary artery. After $\mathrm{PCl}$, all patients were taken to a coronary intensive care unit. Antiplatelet, anticoagulant, ACEI/ARB, $\beta$-blocker, and statin therapy were administered according to the current guidelines.

The door-to-balloon time was defined as the time interval between admission to the hospital and coronary reperfusion after the application of the first device or balloon. The door-to-needle time, however, was defined as the time interval between the admission to the hospital and the administration of thrombolytic therapy. Re-infarction was defined as a $20 \%$ or more increase in new pathognomonic Q-wave development and troponin values or $0.1 \mathrm{mV}$ ST elevation between two consecutive derivations in ECG in addition to ischaemic chest pain over $20 \mathrm{~min}$. Oral hypoglycaemic agent at the time of admission or history of insulin therapy was considered as a diagnosis of diabetes mellitus. Hyperlipidaemia was defined as having an antihyperlipidaemic drug use history or having a total cholesterol level of $200 \mathrm{mg} / \mathrm{dl}$. Hypertension was defined as high blood pressure requiring use of antihypertensive drugs. Positive family history for coronary artery disease was defined as the presence of coronary artery disease in siblings or parents under 60 years of age. Death from cardiovascular causes was defined as acute myocardial infarction, heart failure, arrhythmia, or unexplained sudden death.

\section{Statistical analysis}

SPSS 20.0 (SPSS Inc., Chicago, IL) software was used for statistical analysis of the study findings. Numerical data obtained from the study were presented as mean \pm standard deviation, and the categorical data were expressed as percentages (\%). The $\chi^{2}$ test was used for categorical variables. Student's $t$-test was used for comparison of continuous variables with normal distribution. In all comparisons, $p<0.05$ was considered statistically significant.

Binary logistic regression analysis was performed to indicate independent factors associated with 1-year mortality.

\section{Results}

Our study was conducted with 111 patients over 65 years of age with STEMI $(73$ males, $38 \mathrm{fe}$ males). Of the patients, 66 (59.5\%) were treated with thrombolytic therapy, and 45 (40.5\%) patients underwent primary $\mathrm{PCl}$. The ages of the patients ranged from 65 to 87 years. The mean age of patients in the TT group was $74.17 \pm 5.08$ years, and the mean age of patients in the primary $\mathrm{PCl}$ group was $72.18 \pm 6.77$ years; there was no significant difference between the groups
( $p>0.05)$. Of the patients, $73(65.8 \%)$ were male and 38 (34.2\%) were female. There was no statistically significant difference between the primary $\mathrm{PCl}$ and TT group in terms of gender distribution $(p>0.05)$. There was no statistically significant difference between the two groups in terms of hypertension, diabetes mellitus, smoking, hyperlipidaemia, coronary artery disease history, and family history $(p>0.05)$. Door-to-needle time was $25.9 \pm 7.8 \mathrm{~min}$ in the TT group, whereas door-toballoon time was $84.4 \pm 20.0 \mathrm{~min}$ in the $\mathrm{PCl}$ group. The time interval between the onset of ischaemic chest pain (the most significant symptom of acute myocardial infarction) to hospital admission was $213.6 \pm 158.4 \mathrm{~min}$ in the thrombolytic group and $166.8 \pm 112.8 \mathrm{~min}$ in the $\mathrm{PCl}$ group, and there was no statistically significant difference between the two groups $(p>0.05)$. Rescue PCl was performed in $7(10.6 \%)$ patients in the TT group due to lack of reperfusion. In the TT group, 35 (53\%) patients had anterior MI, whereas 21 (46.7\%) patients in the primary $\mathrm{PCl}$ group had anterior MI (Table I). Table I shows the demographic and clinical characteristics of both groups.

Recurrent infarction was observed in 5 (7.6\%) patients in the TT group and 2 (4.4\%) patients in the $\mathrm{PCl}$ group. Non-haemorrhagic stroke was observed in 1 (1.5\%) patient in the thrombolytic-administered group and in 4 (8.9\%) patients in the $\mathrm{PCl}$ group. No intracranial haemorrhage was seen in any of the patients included in the study. Major haemorrhage was seen in $4(6.1 \%)$ patients in the TT group and in 4 (8.9\%) patients in the primary $\mathrm{PCl}$ group. The major haemorrhages in the TT group were of gastrointestinal system origin, whereas in the primary $\mathrm{PCl}$ group there were two gastrointestinal haemorrhage cases and two haemorrhage cases due to intervention location. There was no significant difference between the groups in terms of recurrent infarction, non-haemorrhagic stroke, major bleeding, and mortality in the inpatient follow-up after both applied reperfusion therapies ( $p>0.05$ ). When the 6-month mortality rates of the patients were taken into account, mortality was observed in 15 (22.7\%) patients in the TT group and in 8 (17.8\%) patients in the primary $\mathrm{PCl}$ group, and there was no statistically significant difference between the two groups $(p>0.05)$. Considering the 1-year mortality rates, mortality was observed in $19(28.8 \%)$ patients in the TT group and in $8(17.8 \%)$ patients in the primary $\mathrm{PCl}$ group. Although 1-year mortality was higher in the TT group, it was not statistically significant $(p>0.05)$. Patients' in-hospital outcomes, and 6-month and 1-year mortality are shown in Table II. Binary logistic regression analysis was performed to indicate independent factors associated with 1-year mortality. Age, gender, 
Table I. Demographic and clinical characteristics of the patients

\begin{tabular}{|c|c|c|c|c|}
\hline Parameter & $\begin{array}{l}\text { All patients } \\
(n=111)\end{array}$ & $\begin{array}{l}\text { Thrombolytic } \\
\quad(n=66)\end{array}$ & $\begin{array}{l}\text { Primary } \mathrm{PCl} \\
\quad(n=45)\end{array}$ & $P$-value \\
\hline Age & $73.4 \pm 5.9$ & $74.2 \pm 5.1$ & $72.2 \pm 6.8$ & 0.080 \\
\hline Female gender, $n(\%)$ & $38(34.2)$ & $22(33.3)$ & $16(35.5)$ & 0.809 \\
\hline Diabetes mellitus, $n$ (\%) & $28(25.2)$ & $16(24.2)$ & $12(26.7)$ & 0.773 \\
\hline Hypertension, $n$ (\%) & $62(55.8)$ & $33(50.0)$ & $29(64.4)$ & 0.132 \\
\hline Hyperlipidaemia, $n$ (\%) & $43(38.7)$ & $27(40.9)$ & $16(14.4)$ & 0.570 \\
\hline Smoking, $n(\%)$ & $19(17.1)$ & $11(16.7)$ & $8(17.8)$ & 0.879 \\
\hline Family history, $n$ (\%) & $28(25.2)$ & $15(22.7)$ & $13(28.9)$ & 0.463 \\
\hline $\begin{array}{l}\text { Time between symptom onset } \\
\text { and admission [min] }\end{array}$ & $194.4 \pm 142.8$ & $213.6 \pm 158.4$ & $166.8 \pm 112.8$ & 0.089 \\
\hline Door-to-balloon time [min] & - & - & $84.4 \pm 20.0$ & - \\
\hline Door-to-needle time [min] & - & $25.9 \pm 7.8$ & - & - \\
\hline Anterior MI, $n(\%)$ & $56(50.4)$ & $35(53.0)$ & $21(46.7)$ & 0.975 \\
\hline
\end{tabular}

Table II. Patients' in-hospital outcomes, and 6-month and 1-year mortality

\begin{tabular}{|lcccc|}
\hline Parameter & $\begin{array}{c}\text { All patients } \\
(n=111)\end{array}$ & $\begin{array}{c}\text { Thrombolytic } \\
(n=66)\end{array}$ & $\begin{array}{c}\text { Primary PCI } \\
(n=45)\end{array}$ & $P$-value \\
\hline In-hospital mortality, $n(\%)$ & $13(11.7)$ & $8(12.1)$ & $5(11.1)$ & 0.871 \\
\hline Recurrent infarction, $n(\%)$ & $7(6.3)$ & $5(7.6)$ & $2(4.4)$ & $4(8.9)$ \\
\hline Non-haemorrhagic stroke, $n(\%)$ & $5(4.5)$ & $1(1.5)$ & $4(8.9)$ & 0.066 \\
\hline Major bleeding, $n(\%)$ & $8(7.2)$ & $4(6.1)$ & $8(17.8)$ & 0.572 \\
\hline 6-Month mortality, $n(\%)$ & $23(20.7)$ & $15(22.7)$ & $8(17.8)$ & 0.184 \\
\hline 1-Year mortality, $n(\%)$ & $27(24.3)$ & $19(28.8)$ & & 0.528 \\
\hline
\end{tabular}

presence of DM, localisation of MI, type of therapy ( $\mathrm{PCl}$ vs. TT), and time from symptom onset to admission were entered into the equation. Age was found to be the only predictor for 1-year mortality (odds ratio (OR): $1.1,95 \%$ confidence interval $(\mathrm{Cl})$ : $1.019-1.188, p=0.015)$.

\section{Discussion}

In this study, we investigated the effects of primary PCl and TT on in-hospital adverse events, and in-hospital and long-term mortality, in patients over 65 years of age with acute STEMI. According to the results of our study, TT and primary $\mathrm{PCI}$ have similar effects in STEMI patients aged 65 years and over, considering the in-hospital adverse outcomes, in-hospital mortality, and 6-month and 1-year mortality rates.

The elderly population is increasing worldwide. Elderly patients occupy account for a large proportion of patients admitted to Emergency Departments due to STEMI. The fact that the elderly population is excluded in most clinical trials suggests that it is necessary to investigate the efficacy and safety of TT and primary PCl practice for this age group. The treatment method to be chosen in this group is important because of the inadequate and contradictory studies performed in this age group, comorbid conditions increasing with age, and high mortality in the elderly [10].

Subgroup analysis of several studies has shown that TT reduces mortality rates in elderly patients compared with conservative treatment [11, 12]. Increased stroke incidence increasing with age and increased intracranial haemorrhage due to age-related amyloid angiopathy are the most feared adverse effects of thrombolytic therapy. In our study, no intracranial haemorrhage was observed in any of the 66 patients aged 65 years and over who received TT. In randomised controlled trials and in case-control studies, it has been shown that there is a greater risk of cerebral haemorrhage than t-PA and streptokinase in the elderly [13]. Despite all risks, thrombolytics increase the survival rate in elderly patients with STEMI [14]. The FTT (Fibrinolytic Therapy Trialists) study group 
showed a greater reduction in absolute mortality rates in patients aged 75 years and older treated with thrombolytics compared to younger patients [11]. Again, the analysis showed a significant relative risk reduction of up to $15 \%$ in mortality in the fibrinolytic treatment group aged 75 years and older [12]. In patients older than 85 years, the risk of haemorrhagic stroke (1.7\%) is significantly lower when compared with 30-day mortality (30\%) [15]. The frequency of haemorrhagic events suggests the necessity of treatment when compared with the possible mortality reduction with fibrinolytic therapy. In a study carried out with patients aged 65 years and older, 30-day mortality rates were found to be $13.5 \%$ in the thrombolytic treatment group, $13 \%$ in the $\mathrm{PCl}$ treatment group, and $20.6 \%$ in the untreated group [16]. In our study, the in-hospital mortality rate was $12.1 \%$ in the TT group and $11.1 \%$ in the primary $\mathrm{PCl}$ group, and 6-month and 1-year mortality rates were $22.7 \%$ and 28.8 in the TT group, and $17.8 \%$ for both periods in the $\mathrm{PCl}$ group. When we look at the in-hospital and 6-month mortality rates, there was no significant difference between the two groups in our study. Although the 1-year mortality rate was higher in the TT group, it was not statistically significant.

In the elderly, primary $\mathrm{PCl}$ is recommended as a reperfusion strategy, which should be preferred for several reasons. First, primary $\mathrm{PCl}$ is a more effective and safer reperfusion therapy than TT. In studies, the rate of successful reperfusion has been found to be higher in patients with primary $\mathrm{PCl}$ than in those treated with thrombolytics $[9,14]$. However, contradictions of thrombolytic therapy and its complications are more severe in the elderly. The most frightening complication of thrombolytic therapy is intracranial haemorrhage, which is more common in elderly patients, but is rare in primary $\mathrm{PCl}$ [17]. In our study, intracranial haemorrhage was not detected in either group. In addition, primary $\mathrm{PCl}$ is a complex process and requires complete personnel and catheter lab preparation, which causes a serious waste of time. TT, however, is a treatment option that can be applied at short notice, and the time between pain and treatment is reduced by rapid treatment. In our study, the door-to-needle time was approximately 26 min and the door-to-balloon time was approximately $84 \mathrm{~min}$.

Considering the demographic characteristics of the 111 patients with STEMI included in our study, there was no statistically significant difference between primary $\mathrm{PCl}$ and $\mathrm{TT}$ groups in terms of gender and age distributions. In terms of clinical features, hypertension, diabetes mellitus, smoking, coronary artery disease history, and hyperlipidaemia were similar in both groups. Although the effect of thrombolytic therapy decreases with age, it is superior to conservative treatment. In elderly patients with increased risk due to comorbid conditions, TT provides significant improvement in mortality and morbidity, but due to the risk of haemorrhage, approximately half of these patients cannot be treated with thrombolytic therapy $[18,19]$.

Although there are many studies comparing primary $\mathrm{PCl}$ and thrombolytic therapy, there are no large-scale studies comparing these treatments in elderly patients. The available information is based on subgroup analysis of large-scale studies. In the NRMI-2 trial, primary PCl has been shown to be less risky for in-hospital mortality and non-fatal stroke in patients over 75 years of age [20]. Findings similar to the NRMI-2 study have also been observed in the PAMI study conducted with patients over 65 years of age. In the PAMI study, tPA infusion therapy and angioplasty had been compared in patients admitted with STEMI. There was no significant difference between the two groups $(0.8 \%$ mortality was observed in both groups) when the end-points of death and myocardial infarction were examined in patients under 65 years of age. However, a significant decrease in the angioplasty group was found when the same end-points were observed in patients aged 65 years and over (8.6\% in the angioplasty group and $20.0 \%$ in the thrombolytic group, $p=0.048$ ) [21]. Similarly, in the SENIOR-PAMI study, it was reported that primary $\mathrm{PCl}$ administered in patients over 75 years of age causes a decrease in the rate of mortality and stroke and the combined end-point [22]. As a result of the GRACE and MITRA studies, it was shown that a significant proportion of elderly patients did not receive reperfusion therapy due to thrombolytic treatment contraindications and that $\mathrm{PCl}$ treatment provided a significant beneficial effect compared to conservative treatment $[18,19]$. In the TRIANA study, which was conducted with patients aged 75 years and older, primary $\mathrm{PCl}$ was compared with new-generation thrombolytics tenecteplase, and the results are in favour of primary $\mathrm{PCI}$ [23]. In addition to these studies, in the STREAM study, primary $\mathrm{PCI}$ was compared with tenecteplase, from the new-generation thrombolytics, in patients in the first $3 \mathrm{~h}$ of the onset of symptoms, who could not undergo primary $\mathrm{PCl}$ within the first hour, and thrombolytic therapy was shown to be as effective as primary $\mathrm{PCl}$. In the same study, after observing higher incidence of intracranial haemorrhage in patients over 75 years of age, the tenecteplase dose was halved in elderly patients, and then the incidence of intracranial haemorrhage in the thrombolytic group was found to be similar to that of primary $\mathrm{PCl}$ in this case [24]. The efficacy of the new-gen- 
eration thrombolytic tenecteplase has been found to be superior regarding coronary revascularisation in these trials, but tenecteplase was not used widely in our country in the study period. In the GUSTO-IIB study, conducted with 1138 patients, the patients were randomised to angioplasty and thrombolytic therapy (tPA) groups, and although primary $\mathrm{PCl}$ was found to be superior at 30-day end-points (death, myocardial infarction, stroke), the difference between the two groups was not significant at the same outcome points in 6-month follow-up [10].

Due to the single-centred design of our study, the results cannot be generalised, and hence prospective, multi-centred studies are needed in this regard. The limited number of patients included in the study and its retrospective nature are other limitations of our study. Therefore, the study has all the disadvantages of retrospective research. The new-generation thrombolytics (such as tenecteplase) and novel advances in interventional therapy could affect the outcomes of this study. The study was performed in selected cases (patients with cardiac arrest and cardiogenic shock were excluded), so the outcomes cannot be generalised.

In conclusion, according to the results of our study, primary $\mathrm{PCl}$ and $\mathrm{TT}$ have similar effects on in-hospital, 6-month, and 1-year mortality rates in the treatment of patients with STEMI, who are over 65 years of age, in selected cases. In addition, non-haemorrhagic stroke, major haemorrhage and recurrent infarction rates were found to be similar. Due to increased morbidity and haemorrhage complications in elderly patients, physicians avoid TT. According to the study results, because they have similar reperfusion periods, thrombolytic treatment could be applied in elderly patients in centres without 24-hour service for primary $\mathrm{PCl}$, which requires complex personnel and catheter laboratory preparation.

\section{Conflict of interest}

The authors declare no conflict of interest.

\section{References}

1. Ibanez B, James S, Agewall S, et al. 2017 ESC Guidelines for the management of acute myocardial infarction in patients presenting with ST-segment elevation: The Task Force for the management of acute myocardial infarction in patients presenting with ST-segment elevation of the European Society of Cardiology (ESC). Eur Heart J 2018; 39: 119-77.

2. Mehta RH, Parsons L, Rao SV, Peterson ED; National Registry of Myocardial Infarction (NRMI) Investigators. Association of bleeding and in-hospital mortality in black and white patients with ST-segment elevation myocardial infarction receiving reperfusion. Circulation 2012; 125: 1727-34.
3. Yeh RW, Sidney S, Chandra M, Sorel M, Selby JV, Go AS. Population trends in the incidence and outcomes of acute myocardial infarction. N Engl J Med 2010; 362: 2155-65.

4. Terkelsen CJ, Sørensen JT, Maeng M, et al. System delay and mortality among patients with STEMI treated with primary percutaneous coronary intervention. JAMA 2010; 304: 763-71.

5. Armstrong PW, Boden WE. Reperfusion paradox in ST-segment elevation myocardial infarction. Ann Intern Med 2011; 155: 389-91.

6. Alexander KP, Newby LK, Armstrong PW, et al. Acute coronary care in the elderly, part II: ST-segment-elevation myocardial infarction: a scientific statement for healthcare professionals from the American Heart Association Council on Clinical Cardiology: in Collaboration with the Society of Geriatric Cardiology. Circulation 2007; 115: 2570-89.

7. Haase KK, Schiele R, Wagner S, et al. In-hospital mortality of elderly patients with acute myocardial infarction: data from MITRA (Maximal Individual Therapy in Acute Myocardial Infarction) registry. Clin Cardiol 2000; 23: 831-6.

8. Stone GW, Brodie BR, Griffin JJ, et al. Prospective, multicenter study of the safety and feasibility of primary stenting in acute myocardial infarction: in-hospital and 30-day results of the PAMI stent pilot trial. J Am Coll Cardiol 1998; 31: 23-30.

9. Keeley EC, Boura JA, Grines CL. Primary angioplasty versus intravenous thrombolytic therapy for acute myocardial infarction: a quantitative review of 23 randomised trials. Lancet 2003; 361: 13-20.

10. The Global Use of Strategies to Open Occluded Coronary Arteries in Acute Coronary Syndromes (GUSTO IIb) Angioplasty Substudy Investigators. A clinical trial comparing primary coronary angioplasty with tissue plasminogen activator for acute myocardial infarction. N Engl J Med 1997; 336: 1621-8.

11. Fibrinolytic Therapy Trialists' (FTT) Collaborative Group. Indications for fibrinolytic therapy in suspected acute myocardial infarction: collaborative overview of early mortality and major morbidity results from all randomised trials of more than 1000 patients. Lancet 1994; 343: 311-22.

12. White HD. Thrombolytic therapy in the elderly. Lancet 2000; 356: 2028-30.

13. Gore JM, Granger CB, Simoons ML, et al. Stroke after thrombolysis. Mortality and functional outcomes in the GUSTO-I trial. Global use of strategies to open occluded coronary arteries. Circulation 1995; 92: 2811-8.

14. Berger AK, Schulman KA, Gersh BJ, et al. Primary coronary angioplasty vs thrombolytics for the management of acute myocardial infarction in elderly patients. JAMA 1999; 282: 341-8.

15. Brieger D, Eagle KA, Goodman SG, et al. Acute coronary syndromes without chest pain, an underdiagnosed and undertreated high-risk group. Chest 2004; 126: 461-9.

16. Kandzari DE, Granger CB, Simoons ML, et al. Risk factors for intracranial hemorrhage and nonhemorrhagic stroke after fibrinolytic therapy (from the GUSTO-I Trial). Am J Cardiol 2004; 93: 458-61.

17. Brass LM, Lichtman JH, Wang Y, Gurwitz JH, Radford MJ, Krumholz HM. Intracranial hemorrhage associated with thrombolytic therapy for elderly patients with acute myocardial infarction: results from the cooperative cardiovascular project. Stroke 2000; 31: 1802-11.

18. Zahn R, Schuster S, Schiele R, et al. Comparison of primary angioplasty with conservative therapy in patients 
with acute myocardial infarction and contraindication for thrombolytic therapy. Maximal Individual Therapy In Acute Myocardial Infarction (MITRA) study group. Catheter Cardiovasc Interv 1999; 46: 127-33.

19. Eagle KA, Goodman SG, Avezum A, Budaj A, Sullivan CM, Lopez-Sendon J; GRACE Investigators. Practice variation and missed opportunities for reperfusion in ST-segment-elevation myocardial infarction: findings from the Global Registry of Acute Coronary Events (GRACE). Lancet 2002; 359: 373-7.

20. Canto JG, Zalenski RJ, Ornato JP, et al. Use of emergency medical services in acute myocardial infarction and subsequent quality of care: observations from the National Registry of Myocardial Infarction 2. Circulation 2002; 106: 3018-23.

21. Stone GW, Grines CL, Browne KF, et al. Predictors of in-hospital and 6-month outcome after acute myocardial infarction in the reperfusion era: the Primary Angioplasty in Myocardial Infarction (PAMI) trial. J Am Coll Cardiol 1995; 25: 370-7.

22. Grines C. Senior PAMI: a prospective randomized trial of primary angioplasty and thrombolytic therapy in elderly patients with acute myocardial infarction. Presented at Transcatheter Cardiovascular Therapeutics; Washington, D; October 16-21, 2005.

23. Bueno H, Betriu A, Heras M, et al.; TRIANA Investigators. Primary angioplasty vs. fibrinolysis in very old patients with acute myocardial infarction: TRIANA (TRatamiento del Infarto Agudo de miocardio eN Ancianos) randomized trial and pooled analysis with previous studies. Eur Heart J 2011; 32: 51-60.

24. Armstrong PW, Gershlick AH, Goldstein P, et al. Fibrinolysis or primary $\mathrm{PCl}$ in ST-segment elevation myocardial infarction. N Engl J Med 2013; 368: 1379-87. 